\title{
Risk stratification based on J-ACCESS risk models with myocardial perfusion imaging: Risk versus outcomes of patients with chronic kidney disease
}

\author{
Kenichi Nakajima, MD, PhD, ${ }^{\text {a }}$ Satoko Nakamura, $M D, P h D,{ }^{\text {b,c }}$ Hiroki Hase, MD, \\ PhD, ${ }^{d}$ Yasuchika Takeishi, MD, PhD, ${ }^{\text {e }}$ Shigeyuki Nishimura, MD, PhD, ${ }^{f}$ \\ Yuhei Kawano, MD, PhD, ${ }^{\text {b,g }}$ Tsunehiko Nishimura, MD, PhD ${ }^{\mathrm{h}}$ \\ a Department of Nuclear Medicine, Kanazawa University Hospital, Kanazawa, Japan \\ b Division of Hypertension and Nephrology, National Cerebral and Cardiovascular Center, Suita, \\ Japan \\ c Kansai University of Welfare Sciences, Kashihara, Japan \\ d Department of Nephrology, Toho University Ohashi Medical Center, Tokyo, Japan \\ e Department of Cardiovascular Medicine, Fukushima Medical University, Fukushima, Japan \\ f Saitama Medical University International Medical Center, Hidaka, Japan \\ g Department of Medical Technology, Teikyo University, Fukuoka, Japan \\ h Graduate School of Medical Science, Kyoto Prefectural University of Medicine, Kyoto, Japan
}

Received Apr 4, 2018; accepted May 29, 2018

doi: $10.1007 / \mathrm{s} 12350-018-1330-8$

Background. This study aimed to validate the accuracy of major-event risk models created in the multicenter J-ACCESS prognostic study in a new cohort of patients with chronic kidney disease (CKD).

Methods and Results. Three multivariable J-ACCESS risk models were created to predict major cardiac events (cardiac death, non-fatal acute coronary syndrome, and severe heart failure requiring hospitalization): Model 1 , four variables of age, summed stress score, left ventricular ejection fraction and diabetes; Model 2 with five variables including estimated glomerular filtration rate (eGFR, continuous); and Model 3 with categorical eGFR. The validation data used three-year (3y) cohort of patients with CKD $(n=526$, major events $11.2 \%)$. Survival analysis of low $(<3 \% / 3 y)$, intermediate $(3 \%$ to $9 \% / 3 y)$, and high $(>9 \% / 3 y)$-risk groups showed good stratification by all three models (actual event rates: 3.1\%, 9.9\%, and $15.9 \%$ in the three groups with eGFR $\geq 15 \mathrm{~mL} / \mathrm{min} / 1.73 \mathrm{~m}^{2}, P=.0087$ (Model 2). However, actual event rates were equally high across all risk groups of patients with eGFR $<15 \mathrm{~mL} / \mathrm{min} /$ $1.73 \mathrm{~m}^{2}$.

Conclusion. The J-ACCESS risk models can stratify patients with CKD and eGFR $\geq 15$ $\mathrm{mL} / \mathrm{min} / 1.73 \mathrm{~m}^{2}$, but patients with $\mathrm{eGFR}<15 \mathrm{~mL} / \mathrm{min} / 1.73 \mathrm{~m}^{2}$ are potentially at high risk regardless of estimated risk values. (J Nucl Cardiol 2020;27:41-50.)

Key Words: Prognosis $\cdot$ cardiac events $\cdot$ multivariable logistic analysis $\cdot$ heart failure $\cdot \mathrm{C}$ reactive protein

Electronic supplementary material The online version of this article (https://doi.org/10.1007/s12350-018-1330-8) contains supplementary material, which is available to authorized users.

The authors of this article have provided a PowerPoint file, available for download at SpringerLink, which summarizes the contents of the paper and is free for re-use at meetings and presentations. Search for the article DOI on SpringerLink.com.

Funding This study was supported by grants from the Japan Cardiovascular Research Foundation.
Reprint requests: Tsunehiko Nishimura, MD, PhD, Graduate School of Medical Science, Kyoto Prefectural University of Medicine, 465 Kajiicho, Kawara-machi Hirokoji, Kamigyo-ku, Kyoto 602-8566, Japan; nisimura@koto.kpu-m.ac.jp $1071-3581 / \$ 34.00$

Copyright (c) 2018 The Author(s) 


\begin{tabular}{|ll|}
\hline Abbreviations & \\
CAD & Coronary artery disease \\
CKD & Chronic kidney disease \\
eGFR & Estimated glomerular filtration rate \\
HF & Heart failure \\
LVEF & Left ventricular ejection fraction \\
MI & Myocardial infarction \\
SSS & Summed stress score \\
\hline
\end{tabular}

See related editorial, pp. 51-52

\section{INTRODUCTION}

Myocardial perfusion abnormalities and left ventricular (LV) dysfunction can predict major cardiac events, and single-photon emission-computed tomography (SPECT) myocardial perfusion imaging (MPI) can provide vital information for prognosis. ${ }^{1-4}$ Large-scale single- and multi-center studies have found that a larger defect size on MPI, LV dysfunction such as lower LV ejection fraction (LVEF) and higher LV volumes are generally associated with an increased incidence of cardiovascular events. Therefore, these factors have been included as statistically significant variables in uniand multi-variable analyses even in Japanese multicenter cohort studies. $^{5-7}$ In addition, since associated conditions such as diabetes and chronic kidney disease (CKD) are also major predictors of cardiovascular events, the estimation of clinical risk factors could play an important role in clinical practice. ${ }^{7,8}$

Risk models for estimating cardiac events have been developed using score-related event risks ${ }^{9}$ and calculations of event risk $(\%) .{ }^{10,11}$ However, although multifactorial effects on cardiovascular events have been demonstrated, few studies have evaluated the accuracy of these multivariable risk models to predict such events in Japanese populations by comparing predicted and actual outcomes.

J-ACCESS prognostic studies have been ongoing in Japan since 2001, and event risk models to estimate major cardiac events, including cardiac death, non-fatal myocardial infarction (MI) and severe heart failure (HF) requiring hospitalization, have been created for clinical applications. ${ }^{10}$ These J-ACCESS risk models incorporated the risk factors of age, LVEF, and perfusion defects under stress determined using summed stress score (SSS), diabetes, and subsequently estimated glomerular filtration rates (eGFR). ${ }^{11}$ The J-ACCESS-3 study was an independent multi-center study in Japan in patients with CKD, and three-year outcomes were evaluated in patients with $\mathrm{eGFR}<50 \mathrm{~mL} / \mathrm{min} /$ $1.73 \mathrm{~m}^{2}{ }^{12,13}$
The hypothesis of the present study is that risk estimated using the model from the J-ACCESS study could be in agreement with the actual outcome of the JACCESS-3 study, which was used as a validation population. The present study also aimed to determine the effect of eGFR on associations between risk models and outcomes.

\section{METHODS}

\section{Risk Models Created in J-ACCESS Study}

Table 1 summarizes the demographics of the derivation population in the J-ACCESS study of the risk model. The study group consisted of 4,031 patients who were assessed by ${ }^{99 m}$ Tc-tetrofosmin SPECT MPI. The inclusion criteria were $\geq 20$ years of age and undergoing stress and rest MPI due to suspected or known ischemic heart diseases. Patients with the onset of MI or unstable angina pectoris within 3 months, cardiomyopathy, and heart failure with class III or higher New York Heart Association classification were excluded. Perfusion defects were semi-quantified in a 17-segment model using a scale from 0 (normal) to 4 (absent) for each segment and then summed stress/rest/difference scores (SSS/SRS/SDS) were calculated.

During 3 years of follow-up, a total of 175 (4.3\%) of the patients developed the major events of cardiac death $(n=54$; $1.3 \%)$, non-fatal MI $(n=37 ; 0.9 \%)$ and severe HF requiring hospitalization $(n=93 ; 2.3 \%)$.

Three-year risk of events ( $\mathrm{p}, \% / 3 \mathrm{y}$ ) was calculated using the multivariable logistic model:

$$
\mathrm{p}=100 /\left(1+\operatorname{Exp}\left[-\mathrm{b}(0)+\sum(\mathrm{b}(\mathrm{i}) \times \mathrm{x}(\mathrm{i}))\right]\right)(\%),
$$

where $b(i)$ is a parameter estimate of a predictive variable $\mathrm{x}(\mathrm{i})$. To create the logistic risk models, patients who were censored alive within the 3 year follow-up were not included.

Model 1: four-parameter model without eGFR. Multivariable logistic analysis of the 4,031 individuals showed that SSS (with four categorical variables of $0-3,4-8,9-12$, and $\geq 13$ by a 20 -segment model; revised later to $0-3,4-7,8-11$, and $\geq 12$ by a 17-segment model), LVEF, age, and diabetes were significant variables (Table 2). ${ }^{10}$

Model 2: five-parameter model with continuous eGFR. Subsequent analysis based on patients with information about eGFR $(n=2,395)$ clarified the importance of including CKD or eGFR. Therefore, all coefficients were determined based on similar logistic analysis with five variables (Table 2). ${ }^{11}$ SSS was classified as $0(<8)$ and $1(\geq 8)$.

Model 3: five-parameter model with categorical eGFR and four SSS classes. Model 3 was used in Heart Risk View software [Nihon Medi-Physics, Tokyo, Japan], which was a revision of Model 2 that included categorical variables and was intended for practical application to clinical situations. The model included eGFR $(\mathrm{mL} / \mathrm{min} /$ $\left.1.73 \mathrm{~m}^{2}\right)$ classified as $1 \quad(<30), 2 \quad(\geq 30,<45), \quad 3$ 
Table 1. Demographics of derivation and validation populations for risk models

\begin{tabular}{llll}
\hline & $\begin{array}{c}\text { Derivation } \\
\text { population } \\
\text { Model 1 }\end{array}$ & $\begin{array}{c}\text { Derivation } \\
\text { population } \\
\text { Models 2 and 3 }\end{array}$ & $\begin{array}{c}\text { Validation } \\
\text { population } \\
\text { J-ACCESS-3 (CKD) }\end{array}$ \\
\hline Number & 4,031 & 2,395 & 526 \\
Age (years) & $65.9 \pm 10.1$ & $66.1 \pm 10.0$ & $71.6 \pm 10.9$ \\
Male gender (\%) & $64 \%$ & $64 \%$ & $67 \%$ \\
Typical chest pain (\%) & $46 \%$ & $46 \%$ & $34 \%$ \\
Body mass index (kg/m ${ }^{2}$ ) & $23.7 \pm 3.2$ & $23.7 \pm 3.3$ & $24.1 \pm 4.1$ \\
History of myocardial infarction (\%) & $29 \%$ & $31 \%$ & $0 \%$ \\
History of revascularization (\%) & $36 \%$ & $37 \%$ & $0 \%$ \\
Diabetes mellitus (\%) & $29 \%$ & $33 \%$ & $42 \%$ \\
Hypertension (\%) & $55 \%$ & $56 \%$ & $91 \%$ \\
Dyslipidemia (\%) & $47 \%$ & $51 \%$ & $49 \%$ \\
Current smoking (\%) & $16 \%$ & $16 \%$ & $6 \%$ \\
eGFR (mL/min/1.73 m $^{2}$ ) & Not used & $67.3 \pm 30.7$ & $29.0 \pm 12.8$ \\
Summed stress score & $8.7 \pm 11.2$ & $9.0 \pm 11.5$ & $1.1 \pm 3.8$ \\
Summed rest score & $7.3 \pm 10.6$ & $7.6 \pm 11.0$ & $0.8 \pm 1.8$ \\
Summed difference score & $1.4 \pm 3.8$ & $1.5 \pm 3.8$ & $62 \pm 15$ \\
Left ventricular ejection fraction at & $62 \pm 14$ & $61 \pm 14$ & $91 \pm 39$ \\
$\quad$ rest (\%) & & & $39 \pm 31$ \\
End-diastolic volume at rest $(\mathrm{mL})$ & $85 \pm 36$ & $85 \pm 38$ & 13 \\
End-systolic volume at rest $(\mathrm{mL})$ & $36 \pm 29$ & $37 \pm 31$ & \\
Reference No. & 10 & 11 & \\
\hline CKD & & & \\
\hline
\end{tabular}

$C K D$, chronic kidney disease; $e G F R$, estimated glomerular filtration rate

Table 2. Parameter estimates based on multivariable logistic analysis in three risk models

\begin{tabular}{|c|c|c|c|c|c|c|}
\hline & \multicolumn{2}{|c|}{ Model 1} & \multicolumn{2}{|c|}{ Model 2} & \multicolumn{2}{|c|}{ Model 3} \\
\hline & Coefficients & Comments & Coefficients & Comments & Coefficients & Comments \\
\hline Age & 0.0558 & Continuous & 0.0582 & Continuous & 0.0572 & Continuous \\
\hline $\begin{array}{l}\text { Diabetes } \\
\text { mellitus }\end{array}$ & 0.8858 & Yes (1)/no (0) & 0.7998 & Yes (1)/no (0) & 0.7742 & Yes (1)/no (0) \\
\hline LVEF & -0.0475 & Continuous & -0.0359 & Continuous & -0.0363 & Continuous \\
\hline SSS & 0.1941 & Categorical (0-3) & 0.697 & Categorical $(0,1)$ & 0.2305 & $\begin{array}{c}\text { Categorical } \\
(0-3)\end{array}$ \\
\hline eGFR & - & None & -0.0151 & Continuous & -0.3522 & $\begin{array}{c}\text { Categorical } \\
(1-5)\end{array}$ \\
\hline Intercept & -4.8125 & & -4.699 & & -4.5815 & \\
\hline $\begin{array}{l}\text { Number of } \\
\text { patients } \\
\text { for } \\
\text { creating } \\
\text { models }\end{array}$ & 4,031 & & 2,453 & & 2,453 & \\
\hline
\end{tabular}

eGFR, estimated glomerular filtration rate; $L V E F$, left ventricular ejection fraction; SSS, summed stress score 
$(\geq 45,<60), 4(\geq 60,<90)$, and $5(\geq 90)$; and SSS was classified as $0(\leq 3), 1(4-7), 2(8-11)$, and $3(\geq 12)$.

\section{Patients in the Validation Population}

The principal design and results of J-ACCESS-3 are described elsewhere. ${ }^{14}$ A total of 549 patients were registered from 62 institutions between 2009 and 2010. The inclusion criteria included patients aged $\geq 20$ years who were scheduled to undergo stress-rest electrocardiographic gated MPI due to having suspected ischemic coronary artery disease (CAD), eGFR $<50 \mathrm{~mL} / \mathrm{min} / 1.73 \mathrm{~m}^{2}$, and one or more of the seven risk factors for CAD (hypertension, diabetes, dyslipidemia, peripheral vascular diseases, currently smoking, family history of juvenile $\mathrm{CAD}$, and history of ischemic stroke). Chronic kidney disease was defined based on the Japanese equation for eGFR $\left(\mathrm{mL} / \mathrm{min} / 1.73 \mathrm{~m}^{2}\right){ }^{15}$ The exclusion criteria included hemodialysis or peritoneal dialysis, severe valvular heart disease requiring surgical treatment, cardiomyopathies, prior diagnosis of angina pectoris or MI, and a history of revascularization, percutaneous coronary intervention (PCI), or a coronary artery bypass graft surgery. A total of 526 patients who had complete sets of variables for the risk model were analyzed.

\section{MPI Protocol}

Either 1-day (96\%) or 2-day (4\%) protocols were applied in pharmacological stress MPI studies using a standard protocol of ${ }^{99 \mathrm{~m}} \mathrm{Tc}$-tetrofosmin and Anger-type cameras. Pharmacological stress studies proceeded using adenosine (91\%), dipyridamole, or adenosine triphosphate. The average administered doses of ${ }^{99 \mathrm{~m}} \mathrm{Tc}$-tetrofosmin for the initial and second studies were 312 and $689 \mathrm{MBq}$, respectively. Chest pain $(n=7,1.3 \%)$, ST depression $(n=3,0.6 \%)$, atrioventricular block (the second degree or worse) $(n=2,0.4 \%)$, and serious arrhythmia $(n=2,0.4 \%)$ developed during the pharmacological stress study. Both LVEF and LV volumes were quantified using QGS software (Cedars-Sinai Medical Center, Los Angeles, CA, USA).

\section{Evaluation of MPI Findings}

An interpretation committee comprising seven nuclear cardiology experts objectively analyzed all data from standard SPECT slices including SSS, SRS, and SDS. Thresholds for defect scoring were based on a normal database derived from the Japanese Society of Nuclear Medicine working group database. $^{16}$

\section{Events During Follow-Up}

The endpoints of the J-ACCESS-3 study were major cardiac events comprising cardiac death, sudden cardiac death, non-fatal MI, and hospitalization to treat HF. Sudden death was defined as death of unknown cause at 24 hours after occurrence. These major cardiac events were confirmed at each institution based mainly on medical charts, along with written questionnaires and telephone interviews. During the 3-year follow-up, $4.8 \%$ and $3.6 \%$ of the patients were lost to the JACCESS and J-ACCESS 3 studies, respectively.

\section{Ethical Approval}

The Institutional Review Boards of all participating hospitals approved both the J-ACCESS and J-ACCESS-3 studies, both of which also complied with the Ethical Guidelines for Epidemiological Research in Japan. ${ }^{14}$ All patients provided written informed consent to participate in the study.

\section{Statistical Analysis}

Data are expressed as mean \pm standard deviation (SD) or median and ranges for non-normal distribution. Continuous variables were compared using $T$ tests and analyses of variance. Categorical data between groups were compared using $\chi^{2}$ tests. Since C-reactive protein (CRP) was one of the major independent prognostic factors at a threshold of $0.3 \mathrm{mg}$ / $\mathrm{mL},{ }^{13}$ the frequency of high CRP was also examined in each group. Calculated differences in cardiac event risks among groups within the three models were compared using KaplanMeier survival analysis. Appropriate thresholds for three models were determined from the three risk groups of patients by analyzing receiver-operating characteristics (ROC) curves and histograms; $<3 \% / 3$ years (1\%/year) for low-risk patients and $>9 \%$ for high-risk patients (corresponding to the lower and upper quartiles of the event risks). All data were statistically analyzed using JMP software version 12.2 (SAS Institute Inc., Cary, NC, USA). A $P$ value of $<0.05$ was considered to indicate a significant difference.

\section{RESULTS}

\section{Demographics of Patients}

Table 1 summarizes the background conditions for the model-derivation groups and the J-ACCESS-3 study. The validation population did not include any patients with a history of either MI or revascularization. The eGFR values in Model 2 and J-ACCESS-3 were $67.3 \pm 30.7$ and $29.0 \pm 12.8 \mathrm{~mL} / \mathrm{min} / 1.73 \mathrm{~m}^{2}$ $(P<.0001)$, respectively. The SSS, SRS, and SDS were lower in J-ACCESS-3 than in the Model 2 derivation populations $(P<.0001$ for all $)$.

\section{Major Cardiovascular Events}

Out of 526 patients $59(11.2 \%)$ patients developed cardiac events, and $13(2.5 \%)$ had cardiac or sudden death. The events in these 59 patients comprised HF treated upon admission $(n=42)$ sudden death $(n=6)$, cardiac death $(n=3)$, death due to HF upon admission $(n=3)$, non-fatal MI $(n=2)$, non-fatal MI and HF upon 
admission $(n=2)$, and non-fatal MI and subsequent cardiac death $(n=1)$. We found lower values for LVEF $(52.1 \% \pm 14.8 \%$ vs $62.7 \% \pm 14.7 \%, P<.0001)$ and eGFR $\left(25.0 \pm 12.4\right.$ vs $29.4 \pm 12.7 \mathrm{~mL} / \mathrm{min} / 1.73 \mathrm{~m}^{2}$, $P=.026)$, and higher values for EDV $(113.9 \pm 43.6$ vs $88.1 \pm 38.3 \mathrm{~mL}, \quad P=.0004), \quad$ ESV $(58.1 \pm 35.3$ vs $37.0 \pm 30.2 \mathrm{~mL}, \quad P=.0003), \quad$ SSS $\quad(3.7 \pm 6.7 \quad$ vs $1.6 \pm 3.2, P=.046)$, and SRS $(2.8 \pm 6.0$ vs $0.9 \pm 2.4$, $P=.040)$, in a group that developed HF as the first event $(n=45)$ compared with a group that did not develop events $(n=466)$. The SDS did not significantly differ between these groups $(1.0 \pm 2.1$ vs $0.8 \pm 1.8$, $P=\mathrm{ns})$.

Renal events of hemodialysis and peritoneal dialysis developed in $45(8.6 \%)$ and $4(0.8 \%)$ patients, respectively. Patients with eGFR $<15 \mathrm{~mL} / \mathrm{min} / 1.73 \mathrm{~m}^{2}$ $(n=99)$ showed more frequent renal events including $40(40.4 \%)$ with hemodialysis and 4 (4.0\%) with peritoneal dialysis.

\section{Distribution of Risk Values Calculated by Three Models}

The median risk values were $3.3 \%$ (range $0.2 \%$ $36.6 \%), 5.3 \%(0.25 \%-44.2 \%)$, and $5.0 \%(0.25 \%-44.5 \%)$ for Models 1, 2, and 3, respectively, and were highest for Model 2 (Models 1 vs 2, and 1 vs 3, both $P<.0001$; Model 2 vs $3 P=.0041$ ). High correlation was observed for risk values between Models 1, 2, and 3 showing Risk by Model $2=1.5+1.21 \times$ Risk by Model 1 $\left(R^{2}=0.92, \quad P<.0001\right)$ and Risk by Model $2=0.40+1.03 \times$ Risk by Model $3 \quad\left(R^{2}=0.97\right.$, $P<.0001)$.

\section{Estimation of Cardiac Event Risk by Models 1, 2, and 3 with Respect to eGFR}

Table 2 shows parameter estimates for the three $\mathrm{J}$ ACCESS risk models. ${ }^{10,11}$ Figure 1 graphically shows an example of differences in estimated risk in a 60-year

\section{A Diabetes}

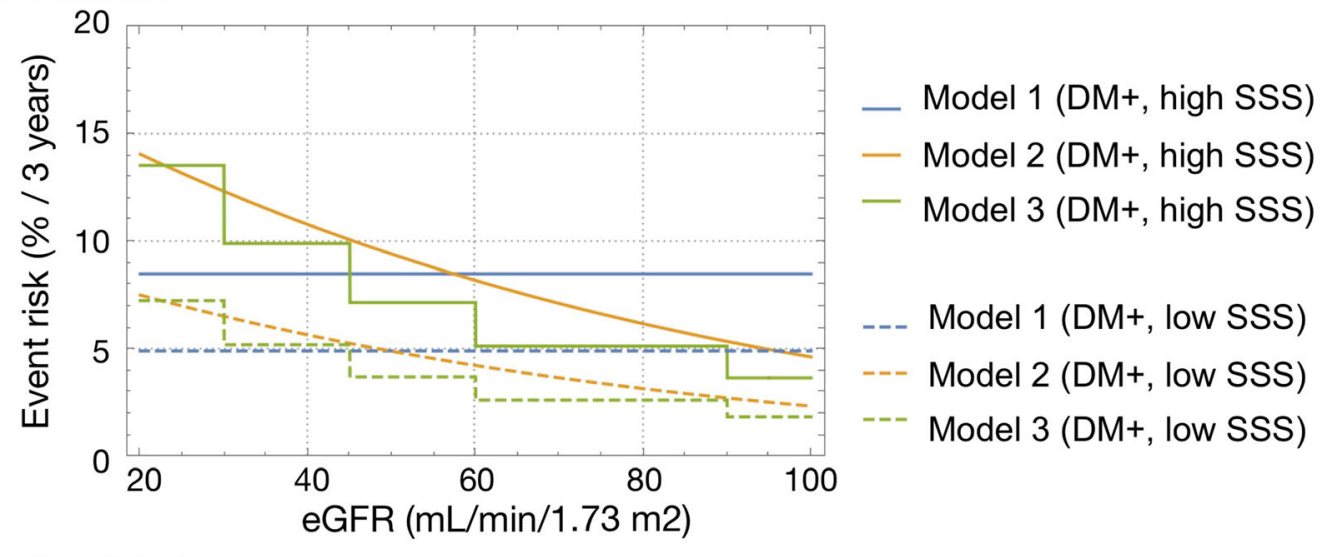

B No diabetes

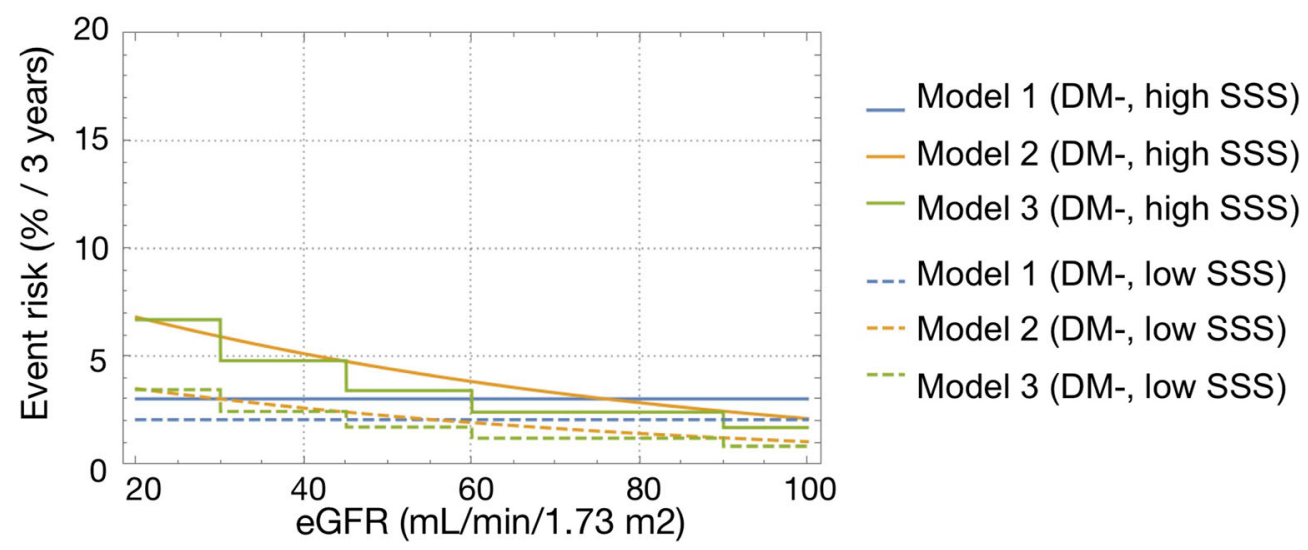

Figure 1. Comparison of three models depending on eGFR in a patient aged 60 years. With (+) and without (-) diabetes (DM), high/low summed stress score (SSS) and left ventricular ejection fraction $50 \%$. 
old patient with LVEF 50\%. Event risk determined by Model 1 was not influenced by the eGFR values defined in the model, whereas calculated risk negatively depended on eGFR in Models 2 and 3. These graphs also indicated that the estimated risk was two-fold higher for patients with high $(>7)$ compared with low $(\leq 7)$ SSS. Furthermore, complication with diabetes mellitus also increased risk two-fold.

\section{ROC Analysis for Predicting Cardiac Events}

Areas under the ROC curve (AUC) were 0.66 (95\% confidence of intervals [CI]: $0.58-0.73), 0.67$ (0.59$0.73)$, and $0.66(0.58-0.73)$ for Models 1, 2, and 3, respectively, indicating that the models similarly predicted low to high major cardiac event. Thresholds were determined from the highest value for sensitivity + specificity -1 at $4.3,5.2$, and $4.5 \%$ risk for Models 1, 2, and 3, respectively.

\section{Survival Analysis and Event Rates in Three Risk Models}

Figure 2 shows that the groups with low $(<3 \% / 3$ $y)$, intermediate $(3 \%-9 \% / 3 y)$ and high $(>9 \% / 3$ y) risk for developing major events significantly differed among Models $1(P=.0001), 2(P=.0014)$, and 3 $(P=.0047)$.

Figure $3 \mathrm{~A}$ shows that actual event rates significantly differed among the three risk groups. Figure 3B shows that when the patients were classified according to an eGFR threshold of $15 \mathrm{~mL} / \mathrm{min} / 1.73 \mathrm{~m}^{2}$, each risk

\section{A Model 1}

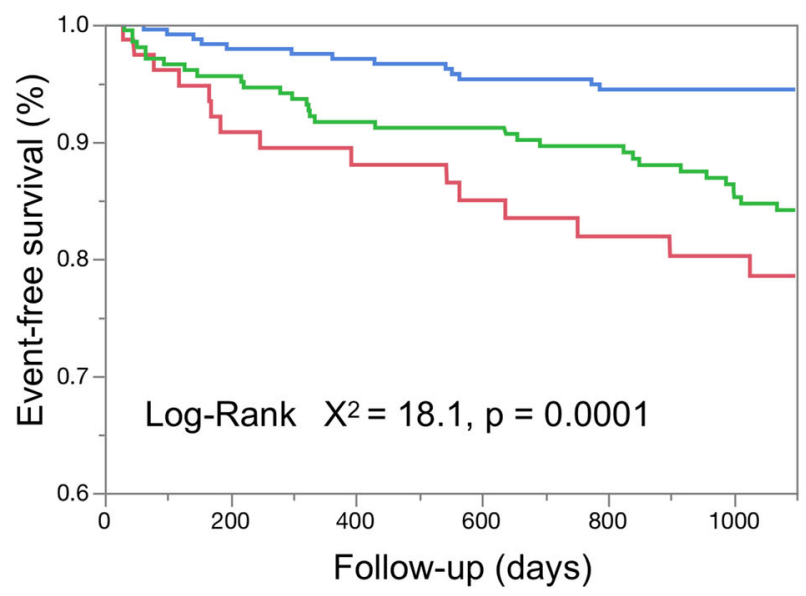

\section{Model 3}

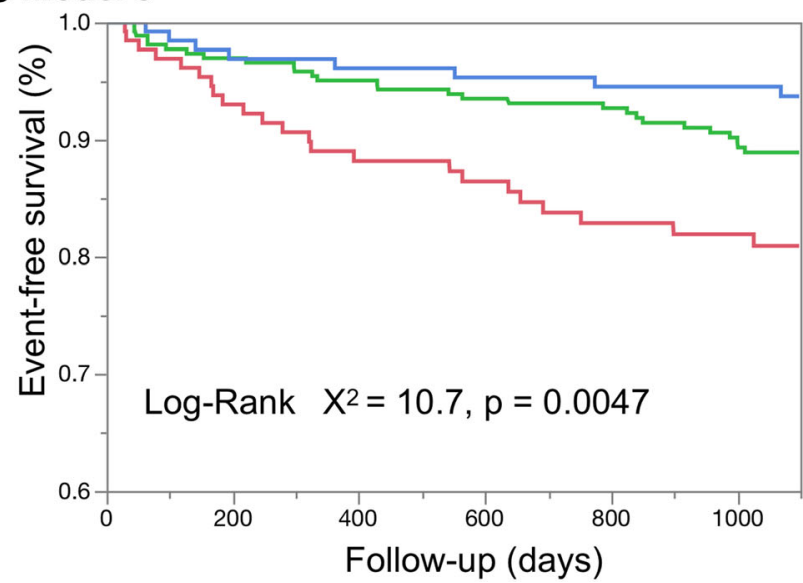

\section{B Model 2}

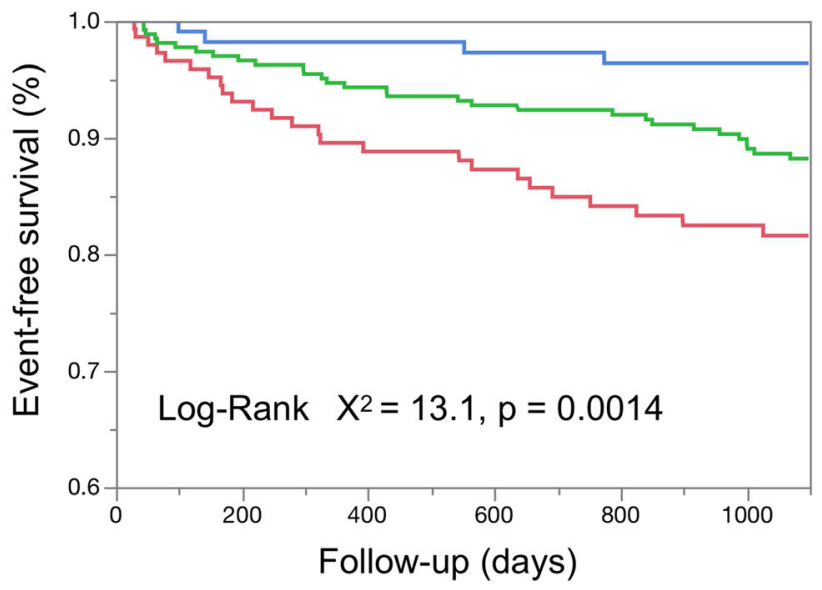

Figure 2. Survival analysis by three multivariable models. Model 1, without eGFR; Model 2, with continuous eGFR value and two SSS categories; Model 3, with categorical eGFR value and four SSS categories. Blue, green and red curves, patients at low $(<3 \% / 3 \mathrm{y})$, intermediate $(3 \%-9 \% / 3 \mathrm{y})$ and high $(>9 \%)$ risk of developing cardiac events. 


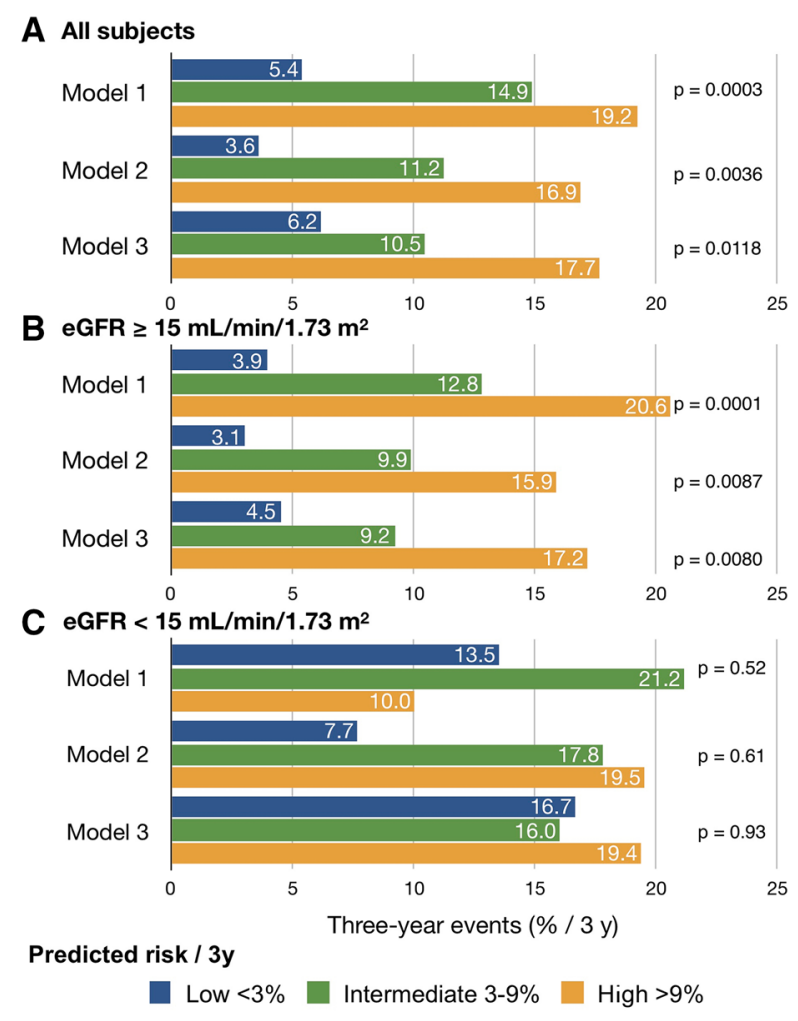

Figure 3. Three-year cardiac event outcome based on three models for patients at low $(<3 \% / 3 \mathrm{y})$, intermediate (3\%-9\%) $3 y)$ and high $(>9 \% / 3 y)$ risk. Panels $(\mathbf{A}),(\mathbf{B})$ and $(\mathbf{C})$ show all patients and those with eGFR $\geq 15$ and $<15 \mathrm{~mL} / \mathrm{min} /$ $1.73 \mathrm{~m}^{2}$, respectively.

group was clearly separated by the three models among those with eGFR $\geq 15 \mathrm{~mL} / \mathrm{min} / 1.73 \mathrm{~m}^{2}$. The actual event rate was the lowest among the low-risk $(<3 \%)$ patients in Model 2 (3.1\%). In contrast, none of the three models could stratify patients with eGFR $<15 \mathrm{~mL} / \mathrm{min} /$ $1.73 \mathrm{~m}^{2}(n=99)$ into three risk groups (Figure 3C).

\section{Frequency of High CRP in Risk Groups}

The CRP values ranged from 0 to 10.1 (median, 0.1 ; mean, $0.42 \pm 0.98) \mathrm{mg} / \mathrm{dL}$. Analyses of ROC curves showed that the optimal cutoff to obtain the highest sensitivity + specificity -1 was $0.3 \quad$ (AUC $=0.62$, $P=0.0029$ ). Figure 4 shows the frequency of high CRP ( $>0.3 \mathrm{mg} / \mathrm{mL}$ ) among patients at low, intermediate, and high risk using Models 1, 2, and 3. The frequency of high CRP was increased among patients with eGFR $\geq 15 \mathrm{~mL} / \mathrm{min} / 1.73 \mathrm{~m}^{2}$ depending on risk for all models $(P=.0004, .0003$, and .0045 for Models 1,2 , and 3, respectively). However, the frequency of elevated CRP was high in all groups of patients with eGFR $<$ $15 \mathrm{~mL} / \mathrm{min} / 1.73 \mathrm{~m}^{2}$ regardless of model type.

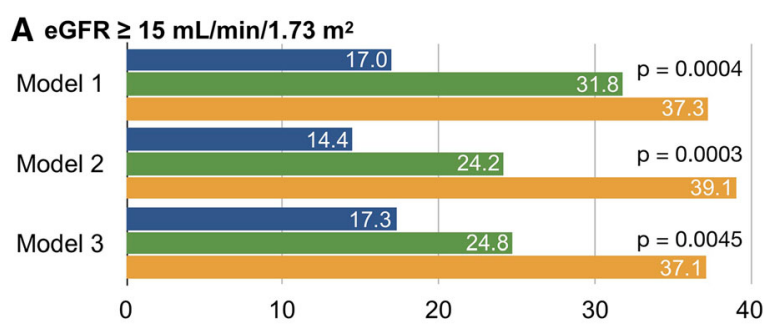

$B$ eGFR $<15 \mathrm{~mL} / \mathrm{min} / 1.73 \mathrm{~m}^{2}$

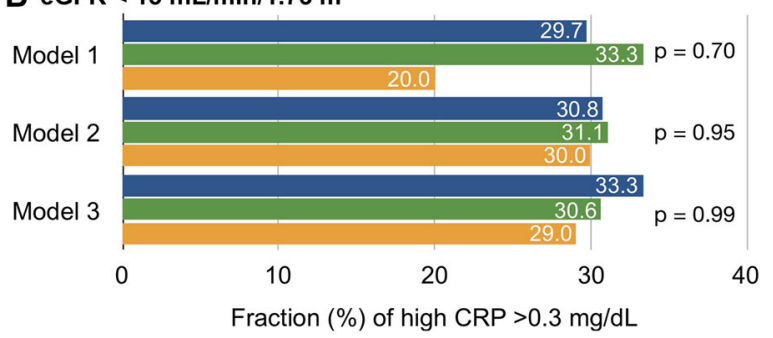

Predicted risk / $3 y$

Low $<3 \%$ Intermediate $3-9 \%$ High $>9 \%$

Figure 4. Frequency of high $\mathrm{CRP}(>0.3 \mathrm{mg} / \mathrm{mL})$ determined in three models among patients at low $(<3 \% / 3 \mathrm{y})$, intermediate (3\%-9\%/3y) and high ( $>9 \% / 3 \mathrm{y}$ risk of developing cardiac events. Panels $\mathrm{A}$ and $\mathrm{B}$, patients with eGFR $\geq 15$ and $<15$ $\mathrm{mL} / \mathrm{min} / 1.73 \mathrm{~m}^{2}$, respectively.

\section{DISCUSSION}

The present study demonstrated the validity of the event risk models created by the J-ACCESS multi-center cohort study and presently applied in Japan. ${ }^{10,11}$ The estimation of major cardiovascular events has practical roles for deciding optimal treatment strategies for patients with suspected CAD. ${ }^{1,3,17}$ Actual event rates were higher than the risk predicted by the model created without eGFR but including eGFR improved risk assessment by the models. However, the incidence of cardiac events was higher regardless of predicted risk in patients with eGFR $<15 \mathrm{~mL} / \mathrm{min} / 1.73 \mathrm{~m}^{2}$, indicating a limitation of the risk model for such patients.

\section{J-ACCESS Risk Model}

The J-ACCESS risk model validated herein was the first risk model created in Japan to estimate major cardiac events using quantitative gated stress-rest MPI. ${ }^{18}$ Based on multivariable logistic analysis, the model included the four variables of age, LVEF, SSS, and diabetes as predictor, ${ }^{10}$ and eGFR was subsequently added. ${ }^{8,11}$ Because SDS did not reach statistical significance in the multivariable analysis, it was not adopted in the model. Since respectively categorizing SSS and eGFR into four and five classes was considered clinically practical, risk calculation software that included these categorical variables was also created for clinical 
applications. The uniqueness of these risk models was that a multivariable logistics model provided major cardiac event risks (\%) for 3 years. Therefore, agreement between the predicted risk and actual event rates was a concern and required validation. The modelderivation and validation populations differed insofar as the latter group had lower eGFR, SSS, and SRS. That is, our model was applied to patients with different demographics. The safety of pharmacological stress in patients with CKD was also confirmed.

\section{Validity of the Risk Models}

The validation study showed that any of the three J-ACCESS risk models could stratify patients into being at low, intermediate, or high risk of developing major cardiac events. However, another important aspect of the model was the identical predicted and actual event rates $(\%)$. The risk of events predicted by Model 1 was underestimated compared with actual event rates (1\%-3\% vs $5.4 \%$ and $3 \%-9 \%$ vs $14.9 \%)$, whereas Model 2 including eGFR predicted 1\%-3\% and $3 \%-9 \%$ risk of events, compared with the actual rates of $3.1 \%$ and $9.9 \%$, respectively. These outcomes were still slightly higher than the upper limit of the predicted values with eGFR. Although Models 2 and 3 tended to be similar, the risk of events predicted by Model 2 was $3.1 \%$, which was lower than the $4.5 \%$ predicted by Model 3 for low-risk patients. Therefore, although the J-ACCESS risk model including eGFR improved risk estimation (\%/3 years), the actual outcomes for patients with CKD remained underestimated to some degree.

\section{Estimated GFR as a Predictor of the Model}

Predictive accuracy was quite different depending on eGFR $>15$ (CKD stages $3 \mathrm{a}, 3 \mathrm{~b}$ and 4 in the present study) or $<15 \mathrm{~mL} / \mathrm{min} / 1.73 \mathrm{~m}^{2}$ (stage 5 ), which was selected because this threshold is a significant predictor of survival in patients with CKD. ${ }^{13}$ Although the actual event rate was near the upper limit or slightly higher than the predicted rates in patients with $\geq 15 \mathrm{~mL} / \mathrm{min} /$ $1.73 \mathrm{~m}^{2}$, the rates were $3 \%-5 \%$ and $9 \%-13 \%$ for lowand intermediate-risk patients, respectively, whose predicted risk was apparently underestimated when eGFR was $<15 \mathrm{~mL} / \mathrm{min} / 1.73 \mathrm{~m}^{2}$. A comparison of Models 2 and 3 showed that Model 2 estimated lower risk for lowrisk patients, and that the difference in outcome events among the risk groups tended to be larger than that of Model 3. This could be explained by differences between the numerical and categorical applications of variables; eGFR $<30 \mathrm{~mL} / \mathrm{min} / 1.73 \mathrm{~m}^{2}$ was categorized into one category in Model 3, but continuous in Model
2. These model structures might have caused the larger difference in calculated risk at lower eGFR.

\section{High-Risk Patients with Low eGFR}

The underestimated risk among patients with CKD stage $5\left(\mathrm{eGFR}<15 \mathrm{~mL} / \mathrm{min} / 1.73 \mathrm{~m}^{2}\right)$ is an important limitation of the present risk model. Since few patients (3\%) had stage $5 \mathrm{CKD}$ (end-stage) in the modelderivation group, the predictive accuracy of the model might be less accurate with respect to this validation group. Although the present study could not determine the pathophysiological reasons for this phenomenon, high CRP or an inflammatory reaction might have been associated, which has also been noted in previous studies. Potentially critical elements in the initiation, progression, and rupture of plaque have become evident, and anti-inflammatory and antioxidant therapies have been sought. ${ }^{19}$ In fact, the distribution of event outcomes and positive CRP were related in the present study (Figures 3, 4), as CRP was elevated at the initiation of dialysis treatment among some patients with CKD and it is a predictor of cardiac events. ${ }^{20,21}$ Analysis of relationships among metabolic syndrome, high-sensitivity CRP, and CKD revealed that CRP is a powerful risk factor for arterial stiffness, cardiovascular events, and mortality. $^{22}$ In the present validation population, the high proportion of patients with severe HF requiring hospitalization might be a result of these complex backgrounds. Moreover, when renal events were analyzed during the 3-year follow-up, 44 of 99 patients required either hemodialysis or peritoneal dialysis. Progression to renal insufficiency could exacerbate cardiac function, leading to chronic HF. However, the long-term effects of hemodialysis are beyond the scope of the present study and will require a follow-up investigation. Another factor might be associated with sympathetic derangement in patients with severe CKD. Cardiac ${ }^{123}$ I-metaiodobenzylguanidine uptake was low among patients with low eGFR, and this contributed to the high cardiac mortality risk among patients with chronic $\mathrm{HF}^{23}$

\section{LIMITATIONS}

The present study included patients with CKD stages 3 to 5 , whereas the model-derivation population included stages 1 to 5 . Although stages 1 and 2 were not included in the validation population, including low-risk patients with an annual event rate of $<1 \%(21 \%$ and $25 \%$ of patients determined by Models 2 and 3, respectively), a wide range of risk was evaluated. A recent validation of J-ACCESS Model 2 also found that an event risk of $10 \%$ separated the prognosis of patients 
with a mean eGFR of $67.4 \pm 24.3 \mathrm{~mL} / \mathrm{min} / 1.73 \mathrm{~m}^{2} .^{24}$ Therefore, although the present study focused on patients with CKD, the J-ACCESS model could apply even to patients with normal eGFR. In addition, further validation studies are needed to clarify the relevance of including additional variables such as CRP to enhance predictive accuracy.

\section{NEW KNOWLEDGE GAINED}

Three J-ACCESS models were applied to assess risk of major cardiac events among CKD patients in the J-ACCESS-3 study. Although all risk models for major cardiac events could stratify outcomes of patients with CKD, the model that included eGFR was more appropriate for these patients. Risk stratification was effective for patients with stages $3 \mathrm{a}, 3 \mathrm{~b}$, and 4 CKD (eGFR $\geq 15$ $\mathrm{mL} / \mathrm{min} / 1.73 \mathrm{~m}^{2}$ ), whereas patients with stage 5 CKD $\left(\right.$ eGFR $\left.<15 \mathrm{~mL} / \mathrm{min} / 1.73 \mathrm{~m}^{2}\right)$ are potentially at high risk across all estimated risk values.

\section{Acknowledgements}

We are grateful to CMIC PMS Co. Ltd., Osaka, for statistical analysis of the data. The authors thank Norma Foster for editorial assistance.

\section{Disclosure}

All authors declare no conflict of interest to disclose.

\section{Open Access}

This article is distributed under the terms of the Creative Commons Attribution 4.0 International License (http:// creativecommons.org/licenses/by/4.0/), which permits unrestricted use, distribution, and reproduction in any medium, provided you give appropriate credit to the original author(s) and the source, provide a link to the Creative Commons license, and indicate if changes were made.

\section{References}

1. Hachamovitch R, Hayes SW, Friedman JD, Cohen I, Berman DS. Comparison of the short-term survival benefit associated with revascularization compared with medical therapy in patients with no prior coronary artery disease undergoing stress myocardial perfusion single photon emission computed tomography. Circulation. 2003;107:2900-7.

2. Shaw LJ, Berman DS, Hendel RC, Alazraki N, Krawczynska E, Borges-Neto $\mathrm{S}$, et al. Cardiovascular disease risk stratification with stress single-photon emission computed tomography technetium$99 \mathrm{~m}$ tetrofosmin imaging in patients with the metabolic syndrome and diabetes mellitus. Am J Cardiol. 2006;97:1538-44.

3. Shaw LJ, Berman DS, Maron DJ, Mancini GB, Hayes SW, Hartigan PM, et al. Optimal medical therapy with or without percutaneous coronary intervention to reduce ischemic burden:
Results from the clinical outcomes utilizing revascularization and aggressive drug evaluation (COURAGE) trial nuclear substudy. Circulation. 2008;117:1283-91.

4. Matsumoto N, Sato Y, Suzuki Y, Kunimasa T, Yoda S, Iida J, et al. Prognostic value of myocardial perfusion single-photon emission computed tomography for the prediction of future cardiac events in a Japanese population: a middle-term follow-up study. Circ J. 2007;71:1580-5.

5. Nishimura T, Nakajima K, Kusuoka H, Yamashina A, Nishimura S. Prognostic study of risk stratification among Japanese patients with ischemic heart disease using gated myocardial perfusion SPECT: J-ACCESS study. Eur $\mathrm{J}$ Nucl Med Mol Imaging. 2008;35:319-28

6. Matsumoto N, Sato Y, Suzuki Y, Kasama S, Nakano Y, Kato M, et al. Incremental prognostic value of cardiac function assessed by ECG-gated myocardial perfusion SPECT for the prediction of future acute coronary syndrome. Circ J. 2008;72:2035-9.

7. Yamasaki Y, Nakajima K, Kusuoka H, Izumi T, Kashiwagi A, Kawamori R, et al. Prognostic value of gated myocardial perfusion imaging for asymptomatic patients with type 2 diabetes: the JACCESS 2 investigation. Diabetes Care. 2010;33:2320-6.

8. Hatta T, Nishimura S, Nishimura T. Prognostic risk stratification of myocardial ischaemia evaluated by gated myocardial perfusion SPECT in patients with chronic kidney disease. Eur J Nucl Med Mol Imaging. 2009;36:1835-41.

9. Hachamovitch R, Hayes SW, Friedman JD, Cohen I, Berman DS. A prognostic score for prediction of cardiac mortality risk after adenosine stress myocardial perfusion scintigraphy. J Am Coll Cardiol. 2005;45:722-9.

10. Nakajima K, Nishimura T. Prognostic table for predicting major cardiac events based on J-ACCESS investigation. Ann Nucl Med. 2008;22:891-7.

11. Nakajima K, Matsuo S, Okuyama C, Hatta T, Tsukamoto K, Nishimura $\mathrm{S}$, et al. Cardiac event risk in Japanese subjects estimated using gated myocardial perfusion imaging, in conjunction with diabetes mellitus and chronic kidney disease. Circ J. 2012;76:168-75.

12. Joki N, Hase H, Kawano Y, Nakamura S, Nakajima K, Hatta T, et al. Myocardial perfusion imaging for predicting cardiac events in Japanese patients with advanced chronic kidney disease: 1-year interim report of the J-ACCESS 3 investigation. Eur J Nucl Med Mol Imaging. 2014;41:1701-9.

13. Nakamura S, Kawano Y, Nakajima K, Hase H, Joki N, Hatta T, et al. Prognostic study of cardiac events in Japanese patients with chronic kidney disease using ECG-gated myocardial Perfusion imaging: Final 3-year report of the J-ACCESS 3 study. J Nucl Cardiol. 2017. https://doi.org/10.1007/s12350-017-0880-5.

14. Nakamura S, Kawano Y, Hase H, Hatta T, Nishimura S, Moroi M, et al. Prognostic study of cardiac and renal events in Japanese patients with chronic kidney disease and cardiovascular risk using myocardial perfusion SPECT: J-ACCESS 3 study design. Ther Apher Dial. 2010;14:379-85.

15. Matsuo S, Imai E, Horio M, Yasuda Y, Tomita K, Nitta K, et al. Revised equations for estimated GFR from serum creatinine in Japan. Am J Kidney Dis. 2009;53:982-92.

16. Nakajima K, Matsumoto N, Kasai T, Matsuo S, Kiso K, Okuda K. Normal values and standardization of parameters in nuclear cardiology: Japanese Society of Nuclear Medicine working group database. Ann Nucl Med. 2016;30:188-99.

17. Boden WE, O'Rourke RA, Teo KK, Hartigan PM, Maron DJ, Kostuk WJ, et al. Optimal medical therapy with or without PCI for stable coronary disease. N Engl J Med. 2007;356:1503-16.

18. Nakajima K, Nishimura T. Cardiovascular events in Japan. Lessons from the J-ACCESS multicenter prognostic study using myocardial perfusion imaging. Circ J. 2012;76:1313-21. 
19. Bhatt DL. Anti-inflammatory agents and antioxidants as a possible "third great wave", in cardiovascular secondary prevention. Am J Cardiol. 2008;101:4D-13D.

20. Hase H, Joki N, Ishikawa H, Fukuda H, Imamura Y, Saijyo T, et al. Prognostic value of stress myocardial perfusion imaging using adenosine triphosphate at the beginning of haemodialysis treatment in patients with end-stage renal disease. Nephrol Dial Transplant. 2004;19:1161-7.

21. Hase H, Joki N, Ishikawa H, Saijyo T, Tanaka Y, Takahashi Y, et al. Independent risk factors for progression of coronary atherosclerosis in hemodialysis patients. Ther Apher Dial. 2006;10:321-7.

22. Tsai SS, Lin YS, Lin CP, Hwang JS, Wu LS, Chu PH. Metabolic syndrome-associated risk factors and high-sensitivity C-reactive protein independently predict arterial stiffness in 9903 subjects with and without chronic kidney disease. Medicine. 2015;94:e1419.

23. Nakajima K, Nakata T, Doi T, Kadokami T, Matsuo S, Konno T, et al. Validation of 2-year 123I-meta-iodobenzylguanidine-based cardiac mortality risk model in chronic heart failure. Eur Heart $\mathbf{J}$ Cardiovasc Imaging. 2018. https://doi.org/10.1093/ehjci/jey016.

24. Aburadani I, Usuda K, Sumiya H, Sakagami S, Kiyokawa H, Matsuo S, et al. Ability of the prognostic model of J-ACCESS study to predict cardiac events in a clinical setting: The APPROACH study. J Cardiol. 2018. https://doi.org/10.1016/j.jjcc. 2017.12.006. 